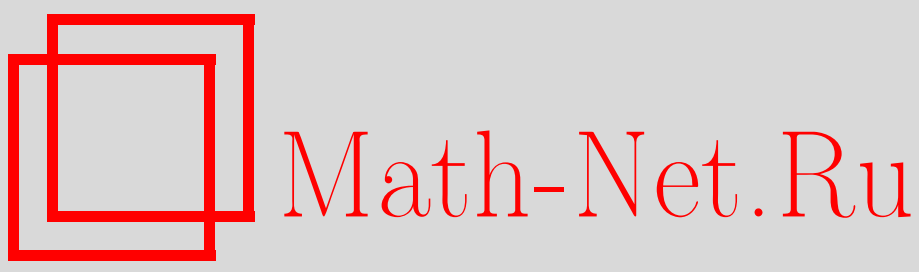

В. А. Акулов, Стохастическая модель отклика системы кровообращения на внешние возмущения, Вестн. Сам. гос. техн. ун-та. Сер. Физ.-мат. науки, 2005, выпуск $38,72-77$

DOI: https://doi.org/10.14498/vsgtu374

Использование Общероссийского математического портала Math-Net.Ru подразумевает, что вы прочитали и согласны с пользовательским соглашением

http://www . mathnet.ru/rus/agreement

Параметры загрузки:

IP : 54.162 .27 .143

26 апреля 2023 г., 11:32:23

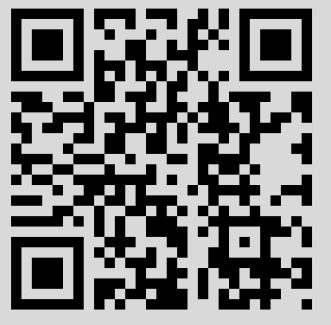




\title{
Биомеханика
}

\author{
УДК $612.001 .573+531.5$ \\ В. А. Акулов

\section{СТОХАСТИЧЕСКАЯ МОДЕЛЬ ОТКЛИКА СИСТЕМЫ КРОВООБРАЩЕНИЯ НА ВНЕШНИЕ ВОЗМУЩЕНИЯ}

\begin{abstract}
Приводятся результаты расчетно-экспериментальных исследований реакции артериального кровотока в магистральных артериях нижних конечностей человека на переход из горизонтального положения в вертикальное (ортостатические пробы). Методами УЗДГ обследовано более пятидесяти здоровых людей, разделенных на подгруппы по возрасту (до 40 лет, более 40 лет) и полу. С помощью непараметрического анализа установлен ряд статистически значимых реакций гемодинамики на ортостатические пробы. В их числе пиковые значения антеградного и ретроградного кровотока в бедренной и подколенной артериях. Выявлен ряд параметров, ответ которых на возмущения незначителен. Исследования выполнены в интересах создания и модернизации аэрокосмических центрифуг как технического средства борьбы с последствиями невесомости и гиподинамии.
\end{abstract}

Широкомасштабные измерения параметров кровотока в артериальном русле конечностей стали возможными благодаря внедрению в медицину методов ультразвуковой допплерографии (УзДГ). Указанные методы являются неинвазивными и позволяют измерить мгновенную скорость крови в сосудах, так называемую линейную скорость кровотока (ЛСК). Исследования, выполненные в интересах авиакосмической медицины, являются весьма специфическими. Сказанное касается как контингента обследуемых, как правило, это здоровые люди, так и номенклатуры решаемых задач. В частности, в статье А. Ю. Модина и В. С. Шашкова [4] приводятся результаты экспериментов по оценке реакции ЛСК на переход человека из горизонтального в вертикальное положение (ортостатические пробы). Оценивая полученные результаты с позиций системного анализа, выделим три важных момента. Нестационарная компонента кровообращения откликается на ортопробы. Ряд параметров показал статистически значимую реакцию. В ряде случаев сдвиги математических ожиданий были весьма значительными, достигая величин $38 \%$. В общей сложности обследовано более 50 практически здоровых людей. Полученные результаты обработаны методами классической статистики ( $t$-критерий Стьюдента). Подчеркнем, что все пациенты, независимо от пола, возраста и других признаков, были сведены в единую (однородную) выборку. Естественным продолжением работ является более детальный анализ с разделением пациентов на подгруппы по указанным признакам, что и сделано ниже.

Острая необходимость в указанной детализации возникла также в процессе решения весьма специфической проблемы «имитационного» моделирования $[1,2]$. Речь идет о применении искусственной силы тяжести в качестве средства профилактики отрицательных последствий длительной гиподинамии и невесомости (пилотируемая космонавтика). Моделируя земную гравитацию, бортовая центрифуга должна воспроизводить, причем в деталях, параметры кровотока. Сказанное в полной мере относится к динамической компоненте кровообращения (ЛСК), соответствующей ортостазу. С одной стороны, указанная компонента является одним из главных объектов имитации. С другой, она изучена крайне недостаточно как с точки зрения медицины, так и проектирования перспективных центрифуг. Так, например, нет ясности в таких вопросах, как необходимость реверсирования, выбор плоскостей вращения и т. д. Важно подчеркнуть, что с имитационным моделирование связан обширный перечень практических приложений. Так, например, представляет интерес оценка значимости отличий в параметрах ЛСК у мужчин и женщин (ортостаз). Если они существенны, то при выполнении профилактических процедур этот фактор должен учитываться, и режимы вращения должны отличаться. В противном случае коррекция режимов по признаку «пол» не требуется, что существенно упрощает процедуры гравитационной терапии. Таким образом, исследование ЛСК представляет собой не только медицинскую, но и промышленную задачу. 
В качестве исходных выбраны те же данные, что и в работе [4]. В результате группировок сформировались выборки малого объема (8-19 наблюдений), которые потребовали перехода от классических методов анализа к непараметрическим $[3,5,6]$. Необходимо отметить, что в медико-технических приложениях они еще не получили должного распространения. Более того, имеются многочисленные примеры их игнорирования и, соответственно, некорректного привлечения классических методов. Изложенное позволяет заключить, что проблема популяризации и внедрения указанных методов является весьма актуальной. Более того, объективные условия таковы, что роль непараметрического анализа в медицине и технике должна неуклонно возрастать. Сошлемся на два очевидных аргумента. Прежде всего, в силу известных экономических причин нередко отсутствуют финансовые, материально-технические и временные ресурсы, достаточные для накопления обширного статистического материала. Очевидно, что в подобных случаях исследователь располагает весьма скромным по объему статистическим материалом (малые выборки). Учитывая изложенные выше технико-медицинские и экономические аспекты, представляется целесообразным решение следующих задач:

1) количественная оценка отклика ЛСК в артериях ног здорового человека на переход из горизонтального в вертикальное положение с распределением пациентов на подгруппы, отличающиеся возрастом (до 40 лет, более сорока лет) и полом;

2) адаптация непараметрического критерия знаков к задачам анализа реакции ЛСК на активные ортостатические пробы;

3) популяризация непараметрических методов в сферах медико-технических исследователей;

4) выдача рекомендаций по результатам анализа и формулировка направлений дальнейших исследований.

Методика. Поскольку проведенные исследования относятся к категории междисциплинарных (медицина - математическое моделирование - информатика), будем различать медицинские и научно-технические аспекты. Что касается медицины, на нее возлагается функция сбора исходных данных (измерение ЛСК средствами УЗДГ) на основе применяемых методик. Научно-технические аспекты включают в себя корректную обработку информации с учетом специфики объекта исследований, формулировку выявленных закономерностей и выдачу соответствующих рекомендаций.

Медицинские аспекты. В ходе экспериментов (А. Ю. Модин, В. С. Шашков, Л. А. Кочергина) выполнялась активная 10-ти минутная ортостатическая проба. В силу специфики решаемых задач из всего многообразия сосудов выбраны артерии нижних конечностей человека: бедренная, подколенная, задняя большеберцовая и тыла стопы. Локализация сосудов осуществлялась из доступов, принятых в практике УЗДГ. Учитывая ограниченный объем статьи, ниже приводятся данные, касающиеся только первых двух доступов, что вполне достаточно для иллюстрации как методических подходов, так и закономерностей внутренней гемодинамики.

Весьма существенным моментом ЛСК, который учтен в настоящих исследованиях, является существование двух противонаправленных потоков крови: антеградного (в прямом направлении) и ретроградного (рис. 1). Для анализа отобраны три общепринятые в практике УЗДГ параметра, а именно: максимальная скорость антеградного, ретроградного кровотоков $\left(V_{\max }^{+}, V_{\max }^{-}\right.$, см. позиции А и В, рис. 1) и индекс сопротивления (ИС).

ИС вычислялся по известной формуле:

$$
\text { ИC }=\left[\left(V_{\max }^{+}\right)+\left(V_{\max }^{-}\right)\right] /\left(V_{\max }^{-}\right) .
$$

Одним из факторов, вносящих некоторую неопределенность в результаты эксперимента, является неравномерное распределение веса по конечностям.

Находясь в вертикальной позе в течение 10 мин, испытуемый поочередно, в произвольные моменты времени, переносит нагрузку с одной ноги на другую. Сказывается также фактор «толчковой» ноги. С целью сведения к

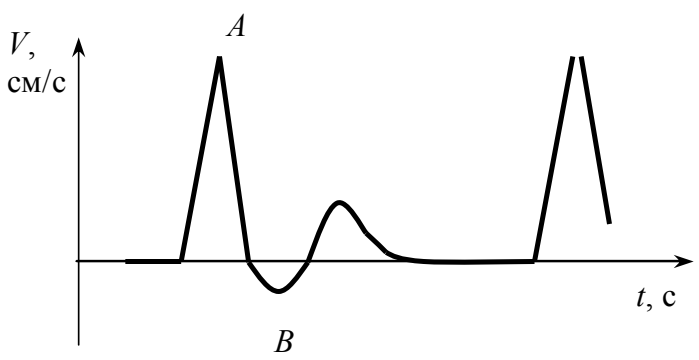

Р и с. 1. Схематическая зависимость мгновенной скорости кровотока в бедренной артерии по времени $(t)$ в течение сердечного цикла минимуму указанной неопределенности анализ ЛСК выполнялся раздельно для каждой из конечностей с последующим сравнением результатов. Подробнее медицинские аспекты изложены в работе [4]. 
Научно-технические аспекты (методические и алгоритмические основы). Результаты медицинских экспериментов послужили исходными данными для научно-технических расчетов (непараметрического анализа). С целью повышения надежности статистических выводов (задача 1) все расчеты были продублированы, что достигалось параллельным применением критерия знаков и знаково-рангового критерия Вилкоксона. В абсолютном большинстве случаев выводы (гипотезы) совпадали. В случаях расхождения принималась гипотеза, выявленная критерием Вилкоксона, как более точным. Вычислялись также показатели описательной статистики $(M, \sigma$, $a)$, где М - математическое ожидание, $\sigma$ - среднее квадратическое отклонение, $a-$ ошибка среднего, которые, как известно, являются стандартными числовыми характеристиками выборок и которые необходимы для продолжения работ. Речь идет о расширении статистического материала с контролем однородности, а также корректном сопоставлении с данными других исследователей.

Учитывая значительный объем информации, ${ }^{1}$ анализ выполнялся в два этапа. Задачей первого этапа была предварительная обработка и визуализация данных, которая заключалась в построении совмещенных графиков, иллюстрирующих отклики параметров на ортопробы. Подобное представление информации представляет значительный интерес для медицинского пользователя. В качестве технического средства применялся критерий знаков, реализованный автором в среде Excel. Окончательная обработка данных (второй этап) выполнялась в профессиональной среде Stadia 6.0 [3] с привлечением обоих упомянутых критериев. В соответствии с формулировками задач 2 и 3, ниже приведены краткие сведения, касающиеся теоретических основ критерия знаков, причем пояснения, комментарии и трактовки адаптированы к исследуемой проблеме. В целях сокращения изложения, критерий Вилкоксона не рассматривается (см., например, [5, 6]).

Исходными данными для моделирования послужили результаты ортостатических проб, представленные в виде парных наборов данных (выборок) вида:

$$
X=\left\{x_{1}, x_{2}, \ldots, x_{n}\right\}, \quad Y=\left\{y_{1}, y_{2}, \ldots, y_{n}\right\} .
$$

Здесь $X$ - исходная выборка, полученная измерением исследуемого параметра (УЗДГ) при горизонтальном положении пациента, а $Y$ - повторная выборка, полученная после перехода в вертикальное положение; $x_{i}, y_{i}$ - результаты измерений соответствующего показателя для $i-$ го пациента; $i=1,2, \ldots, n ; n-$ объем выборок (количество обследованных пациентов).

Например, если $X-$ пиковые скорости $V_{\max }^{+}$, зарегистрированные в горизонтальном положении пациентов в некоторой точке локализации, то $Y$ - соответствующие значение этого же параметра в вертикальном положении. Очевидно, что парные данные $(X, Y)$ содержат количественную информацию о реакции ЛСК пациентов на ортостатические пробы, причем как в индивидуальном порядке, так и группе. Извлечение такой информации, ее представление в виде, удобном для дальнейшего анализа, а главное, интерпретация полученных результатов и является одной из основных задач моделирования. К числу принципиально важных вопросов анализа относится выявление достоверного эффекта воздействия, то есть смещения показателей гемодинамики, под действием возмущений, обусловленных изменением позы или, наоборот, подтверждение его отсутствия. С точки зрения статистики, задача свелась к количественной оценке различия медиан для случая зависимых выборок $X$, $Y$. Если справедлива гипотеза об однородности выборок $\left(H_{0}\right)$, то имеет место соотношение

$$
P\left(x_{i}<y_{i}\right)=P\left(x_{i}>y_{i}\right)=0,5,
$$

где $P(\bullet)$ - вероятность соответствующего события $(\bullet)$. Как следует из (3), число положительных и отрицательных разностей $z_{i}=x_{i}-y_{i}$ (обозначим их соответственно через $S_{+}$и $S_{-}$) не должно значительно отличаться от математического ожидания, равного $n / 2$. Следовательно, справедливо соотношение $S_{+} \approx S_{-}$, а статистический вывод в рамках критерия знаков принимает вид:

$$
\begin{gathered}
\operatorname{Max}\left\{S_{+}, S_{-}\right\}<S_{\kappa p} \Rightarrow H_{0} ; \\
\operatorname{Max}\left\{S_{+}, S_{-}\right\}>=S_{\kappa p} \Rightarrow H_{1},
\end{gathered}
$$

\footnotetext{
${ }^{1}$ Группировки по четырем признакам, три анализируемые параметра ЛСК, четыре точки доступа УЗДГ, две конечности. 
где $H_{1}$ - альтернативная гипотеза: «Имеется статистически значимый эффект воздействия»; $S_{k p}$ - критическое значение, которое зависит от уровня значимости $\alpha$ и находится из таблиц (см., например [5]). В данной работе полагалось $\alpha=0,05$.

Бедренные артерии. На рис. 2 представлено сравнение пиковых значений антеградного кровотока $V_{\max }^{+}$, зарегистрированных в вертикальном положении женщин и мужчин старшего возраста $(49 \pm 7,52 \pm 4$ года соответственно). Обследовано по 12 человек в каждой из подгрупп. Как показал непараметрический анализ с применением обоих критериев, отличия носят случайный характер (гипотеза $H_{0}$, значимость $p \sim 0,34$ ). С практической точки зрения ценность полученного вывода состоит в том, что при выполнении сеансов гравитационной терапии не требуется коррекция режимов вращения ротора ЦКР в зависимости от пола. Это, в свою очередь, существенно упрощает технологию выполнения лечебно-профилактических процедур.

Качественно иной результат получен в отношении реакции кровотока на активные ортостатические пробы по этому же параметру. Как видно из рис. 3 , который иллюстрирует указанную реакцию в группе «мужчины молодого возраста», имеет место стойкое снижение ЛСК. Как показывают расчеты, математическое ожидание уменьшилось по отношению к исходному уровню на $46 \%$ (с 57,25 cм/с до 31,1 см/с, см. табл. 1), что существенно превышает $38 \%$, полученные ранее при укрупненном анализе [4]. Непараметрический анализ данных, представленных на рис. 3, выявил статистически значимые отличия распределений (гипотеза $\mathrm{H}_{1}$ при вероятности $p<0,006)$ по обоим критериям.

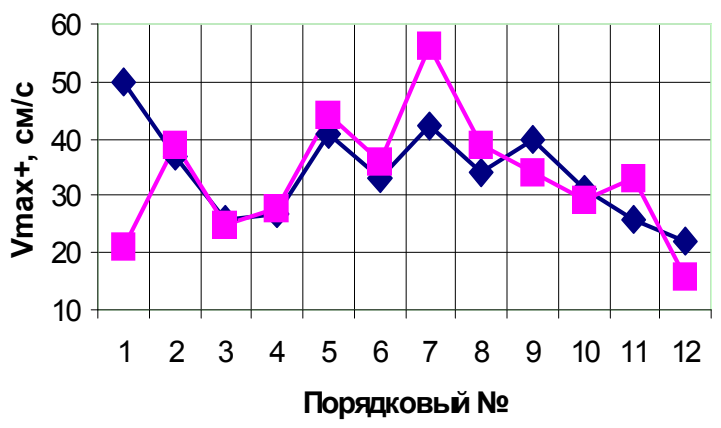

Р и с. 2. Сравнение ЛСК в бедренной артерии по параметру $V_{\max }^{+}$у женщин и мужчин старшего возраста. Средние значения составили соответственно $33,3 \pm 10,7 \mathrm{~cm} / \mathrm{c}, 34,1 \pm 8,2 \mathrm{~cm} / \mathrm{c}$ :

$$
<\text { - женщины, } \mathrm{u} \text { — мужчины }
$$

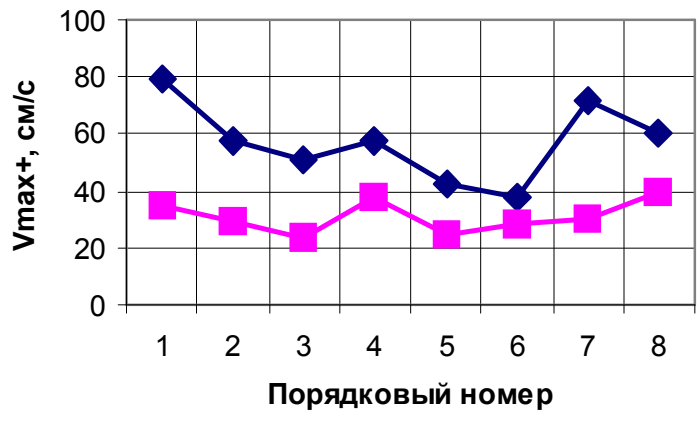

Р и с. 3. Иллюстрация систематического снижения пиковой скорости $V_{\max }^{+}$в бедренной артерии правой ноги при ортостатических пробах. Молодые мужчины (31 \pm 7 лет):

u — горизонтальное положение, < — вертикальное положение

Аналогичный результат получен для других подгрупп пациентов (табл. 1), причем относительное снижение составило 37\% (мужчины старше 40 лет) и 48\% (женщины старше 40 лет). Таким образом, в результате группировок установлено более существенное снижение пиковых значений антеградного кровотока в бедренных артериях в группах «мужчины моложе 40 лет», «женщины старше 40 лет», по сравнению с укрупненным анализом [4]. Исключение составляет группа «мужчины старше 40 лет», данные по которым согласуются с [4].

Непараметрический анализ второго параметра, а им является пиковая скорость ретроградного кровотока $V_{\max }^{-}$, выявляет значимую реакцию на переход к вертикальному положению (гипотеза $\mathrm{H}_{1}$, табл. 1). Подобная закономерность характерна для всех обследованных групп, что несколько расходится с данными [4]. Совершенно очевидно, что указанные выше обстоятельства являются предметом дальнейших исследований.

Третий параметр (ИС (см. (1)) во всех, без исключения, подгруппах незначительно реагировал на пробы (гипотеза $H_{0}$ ), что согласуется с результатами укрупненного анализа [4]. Общая тенденция изменения средней величины ИС в ответ на ортопробы - не убывание. Так, например, в подгруппе мужчины старше 40 лет ИС несколько вырос с 1,34 до 1,4 ( 4,5\%), а в подгруппе женщины старше 40 лет, он практически не изменился $(1,26)$. В то же время, заметно увеличилось рассеивание величины $\sigma$.

Что касается бедренных артерий левой ноги, то обследовано 23 мужчины и 10 женщин. Результаты обработаны в том же объеме, что и для правой ноги. Учитывая качественную иден- 
тичность полученных выводов, подробности, в частности таблицу результатов, не приводим. Очевидно, что полученные результаты требуют всесторонней медицинской оценки.

Т а б ли ц а 1

Отклик параметров ЛСК бедренных артерий на ортостатические пробы

\begin{tabular}{|c|c|c|c|c|c|c|c|c|c|}
\hline Реквизиты & \multicolumn{9}{|c|}{ Бедренная артерия правой ноги } \\
\hline Параметр ЛСК & \multicolumn{3}{|c|}{$V_{\max }^{+}$} & \multicolumn{3}{|c|}{$V_{\max }^{-}$} & \multicolumn{3}{|c|}{ Индекс сопротивления } \\
\hline Пол & \multicolumn{2}{|c|}{$\mathrm{M}$} & Ж & \multicolumn{2}{|c|}{$\mathrm{M}$} & Ж & \multicolumn{2}{|c|}{$\mathrm{M}$} & Ж \\
\hline Возраст, лет & $\leq 40$ & $>40$ & $>40$ & $\leq 40$ & $>40$ & $>40$ & $\leq 40$ & $>40$ & $>40$ \\
\hline Гипотеза & $H_{1}$ & $H_{1}$ & $H_{1}$ & $H_{1}$ & $H_{1}$ & $H_{1}$ & $H_{0}$ & $H_{0}$ & $H_{0}$ \\
\hline $\begin{array}{c}\text { Вероятность по } \\
\text { Вилкоксону } \\
\end{array}$ & 0,006 & 0,0002 & 0,001 & 0,003 & 0,011 & 0,002 & 0,12 & 0,06 & 0,47 \\
\hline $\begin{array}{c}\text { Вероятн. по } \\
\text { кр. знаков }\end{array}$ & 0,0007 & $\sim 0$ & $\sim 0$ & 0,01 & 0,012 & 0,0007 & 0,4 & 0,23 & 0,19 \\
\hline$n$ & 8 & 16 & 12 & 8 & 16 & 12 & 8 & 16 & 12 \\
\hline$M$ & $\begin{array}{c}57,25 / \\
31,1\end{array}$ & $\begin{array}{l}58,1 / \\
36,6\end{array}$ & $\begin{array}{c}64,4 / \\
33,3\end{array}$ & $\begin{array}{c}16,4 / \\
10,1\end{array}$ & $\begin{array}{c}18,7 / \\
14,1\end{array}$ & $\begin{array}{c}16,1 / \\
8,2\end{array}$ & $\begin{array}{l}1,3 / \\
1,32\end{array}$ & $\begin{array}{c}1,34 / \\
1,4\end{array}$ & $\begin{array}{r}1,26 / \\
1,26\end{array}$ \\
\hline$\sigma$ & $\begin{array}{c}13,8 / \\
5,9\end{array}$ & $\begin{array}{l}12,2 / \\
10,8\end{array}$ & $\begin{array}{c}15,8 / \\
10,7\end{array}$ & $\begin{array}{c}8,8 / \\
8,2\end{array}$ & $\begin{array}{c}5,7 / \\
5,3\end{array}$ & $\begin{array}{c}4,9 / \\
5,6\end{array}$ & $\begin{array}{c}0,15 / \\
0,22\end{array}$ & $\begin{array}{c}0,12 / \\
0,14 \\
\end{array}$ & $\begin{array}{c}0,08 / \\
0,16\end{array}$ \\
\hline$a$ & $4,9 / 2,1$ & $3 / 2,7$ & $4,6 / 3,1$ & $3,1 / 2,9$ & $1,4 / 1,3$ & $1,4 / 1,6$ & $0,06 / 0,08$ & $\begin{array}{c}0,03 / \\
0,03\end{array}$ & $\begin{array}{r}0,02 / \\
0,04\end{array}$ \\
\hline
\end{tabular}

Примечания к табл. 1. При дробном представлении результатов (см. $M, \sigma, a)$ в числителе указывается значение, соответствующее горизонтальному положению, в знаменателе - вертикальному.

Подколенная артерия. Результаты обработки данных (непараметрический анализ, описательная статистика, правая нога) представлены в табл. 2. Как видно, закономерности кровообращения в подколенной и бедренной артериях, в основном, идентичны. Как и в предыдущем случае, наблюдаются статистически значимые снижения ЛСК по параметрам $V_{\max }^{+}$и $V_{\max }^{-}$(гипотеза $H_{1}$ ). Так, например, в группе «мужчины старше 40 лет», зарегистрировано снижение скорости $V_{\max }^{+}$при ортопробах с 42,5 $\pm 9,5 \mathrm{~cm} / \mathrm{c}$ до $28,6 \pm 10 \mathrm{~cm} / \mathrm{c}$ или на $33 \%$ по отношению к исходному уровню. Этот же показатель в группе «женщины старше 40 лет» составил 24\%.

Т а б л и ц а 2

Реакция ЛСК подколенной артерии на ортопробы. Мужчины и женщины старше 40 лет ${ }^{2}$

\begin{tabular}{|c|c|c|c|c|c|c|}
\hline Реквизиты & \multicolumn{6}{|c|}{ Подколенная артерия правой ноги } \\
\hline Параметр ЛСК & \multicolumn{2}{|c|}{$V_{\max }^{+}$} & \multicolumn{2}{|c|}{$V_{\max }^{-}$} & \multicolumn{2}{|c|}{ Индекс сопротивления } \\
\hline Пол & $\mathrm{M}$ & Ж & $\mathrm{M}$ & Ж & $\mathrm{M}$ & Ж \\
\hline Гипотеза & $\mathrm{H}_{1}$ & $\mathrm{H}_{1}$ & $\mathrm{H}_{1}$ & $\mathrm{H}_{1}$ & Ho & $\mathrm{H}_{1}$ \\
\hline $\begin{array}{c}\text { Вероятность по } \\
\text { Вилкоксону }\end{array}$ & 0,0001 & 0,0008 & 0,0015 & 0,001 & 0,06 & 0,004 \\
\hline $\begin{array}{l}\text { Вероятность по } \\
\text { критерию знаков }\end{array}$ & 0,0006 & 0,02 & 0,0018 & 0,004 & 0,07 & 0,004 \\
\hline $\mathrm{n}$ & 19 & 10 & 17 & 10 & 17 & 10 \\
\hline$M$ & $\begin{array}{l}42,5 / \\
28,6\end{array}$ & $\begin{array}{l}34,8 / \\
26,3\end{array}$ & $\begin{array}{c}13,1 / \\
6,8\end{array}$ & $\begin{array}{l}9,3 / \\
3,3\end{array}$ & $\begin{array}{l}1,3 / \\
1,4\end{array}$ & $\begin{array}{l}1,28 / \\
1,13\end{array}$ \\
\hline$\sigma$ & $\begin{array}{c}9,5 / \\
10\end{array}$ & $\begin{array}{l}7,1 / \\
4,8\end{array}$ & $\begin{array}{l}5,5 / \\
5,3\end{array}$ & $\begin{array}{c}6,3 / \\
4\end{array}$ & $\begin{array}{l}0,1 / \\
0,9\end{array}$ & $\begin{array}{l}0,17 / \\
0,15\end{array}$ \\
\hline$a$ & $\begin{array}{l}2,2 / \\
2,3\end{array}$ & $\begin{array}{l}2,2 / \\
1,5\end{array}$ & $\begin{array}{l}1,3 / \\
1,3\end{array}$ & $\begin{array}{c}2 / \\
1,3\end{array}$ & $\begin{array}{c}0,02 / \\
0,2 \\
\end{array}$ & $\begin{array}{l}0,05 / \\
0,04\end{array}$ \\
\hline
\end{tabular}

В то же время проявляется некоторая специфика. Прежде всего, заметно уменьшились сами абсолютные величины скорости по этим параметрам как в горизонтальной, так и вертикальной позах. Последнее особенно важно. В частности, для мужчин старшего возраста математическое ожидание $M$ уменьшилось с $36,6 \pm 10,8$ см/с (бедренная артерия, ортостаз, $V_{\max }^{+}$, см.

\footnotetext{
${ }^{2}$ Возраст мужчин 50,2 \pm 6,3 года. Обследовано только 3 мужчины младше 40 лет, поэтому обработка не производилась. Возраст женщин $50 \pm 6$ лет 
табл. 1) до $28,6 \pm 10 \mathrm{~cm} / \mathrm{c}$ (табл. 2), или примерно на $22 \%$, по отношению к исходному уровню. В группе «женщины старше 40 лет» этот показатель составил $21 \%$. Обращает на себя внимание такое обстоятельство, как снижение интенсивности отклика на ортопробы. Так, например, если в бедренной артерии смещение параметра $V_{\max }^{+}$составило 37\% (мужчины старше 40 лет), то в подколенной - 34\%. В группе «женщины старше 40 лет» зарегистрировано более значительное снижение, а именно: с $48 \%$ до $24 \%$, т. е. в два раза. Что касается ИС, в отличие от бедренных артерий, преимущественная реакция на пробы оказалась статистически значимой $\left(H_{1}\right)$. Причем проявилась общая тенденция к убыванию математического ожидания $M$. Указанный вывод уверенно подтверждается обоими критериями в трех исследуемых группах. Исключение составляет выборка «мужчины старше 40 лет, правая подколенная артерия», для которой отклик на ортопробы оказался статистически незначимым, а ИС несколько возрос. Совершенно очевидно, что полученные результаты требуют дополнительных исследований, причем междисциплинарного характера. В частности, известно, что по мере удаления от сердца изменяется структура артерий в стороны увеличения мышечного фактора.

Приведенные выше результаты исследований являются важным шагом к осуществлению натурных экспериментов по имитации динамической компоненты кровообращения во вращающихся системах (центрифуги). Но это отдельная, достаточно сложная научно-техническая задача. На начальных этапах невозможно получение обширного статистического материала, а следовательно, исключается классический статистический анализ. Представляется целесообразным обследование какой-либо одной группы. Настоящая работа предоставляет врачам гравитационной физиологии широкие возможности по выбору контрольной группы.

\section{БИБЛИОГРАФИЧЕСКИЙ СПИСОК}

1. Акулов B. A. Гравитационная терапия: четыре аспекта моделирования гемодинамики конечностей // Вестн. СГАУ, 2004. №1 (5). С. 61-67

2. Котовская А. Р., Шипов А. А., Виль- Вильямс И. Ф. Медико-биологические аспекты проблемы создания искусственной тяжести. М.: Слово, 1986. С. 13-27.

3. Кулаичев А. П. Методы и средства анализа данных в среде Windows Stadia 6.0. М.: НПО «Информатика и компьютеры», 1996. 255 с.

4. Модин А. Ю., Шашков В. С. Влияние гравитации на ЛСК в артериальном русле здорового человека // Авиакосм. и эколог. мед., 2002. Т. 36, № 4. С. 26-29.

5. Рунион Р. Справочник по непараметрической статистике. М.: Финансы и статистика, 1982.

6. Тюрин Ю. Н., Макаров А. А. Анализ данных на компьютере. М.: Финансы и статистика, 1995. 